Abstracted/indexed in Academic Search Complete, Asia Journals Online, Bangladesh Journals Online, Biological Abstracts, BIOSIS Previews, CAB

Abstracts, Current Abstracts, Directory of Open Access Journals, EMBASE/Excerpta Medica, Google Scholar, HINARI (WHO), International

\title{
Preparation and release characteristics study on the novel minocycline hydrochloride sustained-release capsule
}

\author{
Songfeng Zhao', Xiao Zhang', Xiaojian Zhang', Xiuqin Shi', Jun Li ${ }^{1}$, Quancheng Kan² \\ and Shuoye Yang3
}

\author{
${ }^{1}$ Department of Pharmacology, ${ }^{2}$ The Provincial Key Lab of Clinical Medicine, The First Affiliated Hospital of \\ Zhengzhou University, Zhengzhou, Henan 450052, China; ${ }^{3}$ College of Bioengineering, Henan University of \\ Technology, Zhengzhou, China.
}

\begin{tabular}{|c|c|}
\hline Article Info & \\
\hline Received: & 7 January 201 \\
\hline Accepted: & 5 February 201 \\
\hline Available Online: & 21 February 201 \\
\hline DOI: $10.3329 /$ bjp.v & 1 iS1.26415 \\
\hline Cite this article: & \\
\hline Zhao S, Zhang X, & ang $X$, Shi \\
\hline Kan Q, Yang S. T & e preparation \\
\hline $\begin{array}{l}\text { release characteris } \\
\text { novel minocycli }\end{array}$ & $\begin{array}{l}\text { ics study on } \\
\text { e hydrochlor }\end{array}$ \\
\hline ver minocyct & $\begin{array}{l}\text { hydrochloric } \\
\text { se capsule }\end{array}$ \\
\hline Bangladesh J Pha & acol. 2016; 11: S \\
\hline
\end{tabular}

\begin{abstract}
In present study, a novel minocycline hydrochloride sustained-release capsule was prepared with the new extrusion-spheronization method. The in vitro release studies were performed using marketed sample as a reference and data were analyzed in terms of cumulative release amounts as a function of time. Results demonstrated that the developed analysis method was reliable and convenient for the quantification and dissolution study of minocycline hydrochloride. The release characteristics of different batches of preparations were quite similar with each other, similarity factors $f_{2}$ of 12 batches were all within 50-100, and our developed sample was similar to reference preparation in release characteristics in vitro. The developed sustained-release preparation may be a promising alternative dosage form for treatment of related diseases.
\end{abstract}

\section{Introduction}

Minocycline hydrochloride, 4, 7-di-(dimethylamino)- 1, 4, 4a, 5, 5a, 6, 11, 12a-octahydro-3,10,12,12a-tetrahydroxy-1,11-dioxy-2-tetracene methanamide hydrochlorate, is one of the semisynthetic tetracycline derivation antibiotics, which has the potent and sustained antibacterial activity. It has the strongest antibacterial activity among tetracycline antibiotics and the similar antibacterial spectrum compared with tetracycline (Bunagan et al., 2015; Vargiu et al., 2014). It has the inhibitive effects on Gram-positive bacteria, such as Staphylococcus aureus, Streptococcus, and some of the Gram-negative bacteria.

Minocycline exerts a bacterio-static effect by combining to the site A of $30 \mathrm{~S}$ subunit in ribosome, to prevent the extension of peptide chain and inhibit protein synthesis process of bacterium and other pathogenic microorganism (Blanchard et al., 2014). Minocycline is the bacteriostatic drugs and has the bactericidal effect under high concentrations condition (Nagpal et al.,
2015; Norden et al., 2015).

Minocycline hydrochloride is highly lipophilic, has tissue penetration and a range of pharmacological properties such as anti-inflammatory, anti-enzyme and neuroreparable effects (Freiberg et al., 2004; Shibata et al., 2010).

Nowadays, some new theories and methods for sustained or controlled-release are brought about in drug delivery research. Delayed drug delivery system is now the hot topic of oral controlled-release solid dosage forms for pharmaceutical scientists, for instance, the new sustained-release capsule (Peng et al., 2015; Cui et al., 2015). These systems normally consist of a core and a coating. The core is coated with different barriers by film or compression, and the coating can prevent drug quick-release from the core until the shell is completely swollen or eroded by liquid substance in vivo (Ross et al., 2000; Li and Zhu, 2004). These new pharmaceutical preparations can exhibit drug release constantly at a steady rate, the slow and sustained 
release of the active compounds is beneficial to patients to maintain sustainable levels in blood, thus will bring out better compliance to those need long-term and continuous therapy (Liu et al., 2012).

The aim of our study is to develop a novel minocycline hydrochloride sustained-release capsule. In vitro dissolution testing is frequently used to evaluate the release characteristics of the pharmaceutical products over time. On this basis, a release assay method was established and validated to the in vitro release study for sustained-release capsules. The dissolution tests are performed in different media, the dissolution profiles of the commercial and self-made ones are compared by similar factors method, to evaluate the drug release performance of the developed formulation.

\section{Materials and Methods}

\section{Chemicals and reagents}

The reference substances of minocycline hydrochloride (purity $>99.8 \%$ ) was obtained from the National Institute for the Control of Pharmaceutical and Biological Products (China). Potassium dihydrogen phosphate and sodium hydroxide were provided by Qinjiuhong Chemical Reagent Co., Ltd (China). Hydroxypropyl methylcellulose, microcrystalline cellulose and ethyl cellulose were provided by Beijing Huajinsheng Technology Co., Ltd. The commercial product (Razadyne ER) was purchased from the market. Chemicals were purchased from Nanjing Chemical Reagent Co., Ltd (China) and were of analytical grade. Distilled water was purified by a Milli-Q System (Millipore, USA).

\section{Preparation of sustained-release capsule}

In brief, an appropriate quantity of minocycline hydrochloride was weighed and mixed with microcrystalline cellulose, followed by adding a solution of hydroxypropyl methylcellulose in water; pellets were prepared by extruding and rolling. Immediate-release pellets were prepared by using spray-coating technique with the solution of hydroxypropyl methylcellulose on fluidized bed bottom to envelop the sealing coat; some small samples were taken for spraying to coat with ethyl cellulose water dispersion on fluidized bed bottom similarly, to produce sustained-release pellets. Finally, the immediate-release and sustained-release ones were encapsulated to capsules proportionally and packed yielded the products (Dong et al, 2014; Oya et al, 2014).

\section{Development of assay method \\ Specificity}

The reference solution of minocycline hydrochloride, blank preparation (including hydroxypropyl methylcellulose, microcrystalline cellulose, ethyl cellulose, lactose and diethyl phthalate) were taken and diluted by $\mathrm{HCl}$ solution for UV scanning at $200-400 \mathrm{~nm}$.

\section{Linearity}

The developed samples were $19.07 \mathrm{mg}$ standard in minocycline, the appropriate amounts of minocycline hydrochloride was weighed precisely and dissolved in acid solution, to prepare the testing solutions of different concentrations. Absorbencies were determined at $348 \mathrm{~nm}$ for all the samples. The calibration curve samples were assayed in triplicate, using concentration (C) as abscissa and absorbance value (A) as ordinates.

\section{Precision}

Precision was investigated by determining the replicate QC samples of $100 \%$ concentration level in recovery determination experiment on one day and three consecutive days, described as intra-day and inter-day precision, respectively.

\section{Recovery}

Absolute recovery of minocycline hydrochloride was investigated by QC samples, and results were evaluated by comparing the means from the excipients solution spiked with reference solution with that of the standard samples. Three concentration levels of analytes were estimated by analyzing the samples at each level.

\section{Stability}

The stability of minocycline hydrochloride was investigated using the solution at the concentration of $16 \mu \mathrm{g} /$ $\mathrm{mL}$. The samples were analyzed at $0,1,2,3,6$ and 8 hours after conditioning at room temperature, respectively, both in acid solution.

\section{Dissolution assay method for sustained-release capsule}

The oar method for dissolution test was applied to determine dissolution of minocycline hydrochloride from sustained-release capsules. $900 \mathrm{~mL}$ release medium was taken to dissolution glass at predetermined temperature; release medium was agitated by stirring blades at the rotation speed of $100 \mathrm{rpm}$ and sampled at the scheduled time after initiating experiment. $10 \mathrm{~mL}$ sample was collected and filtered through a $0.45 \mu \mathrm{m}$ membrane, filtrate was selected to determine as testing solution.

Minocycline hydrochloride content at each time point was determined by absorbance assay. Meanwhile, the proper amounts of reference substance was dissolved and diluted quantitatively by release medium to the final concentration of $15 \mu \mathrm{g} / \mathrm{mL}$, which was used as standard solution for the total drug amounts (W). The above solutions were analyzed by external standard method, accumulative release amounts and release percent were calculated according to the formula:

$$
Q_{n}=C_{n} V_{0}+\sum_{i=1}^{n-1} C_{i} V_{i} \quad \text { Accumulative release percent }(\%)=Q_{n} / W \times 100 \%
$$


Qn was the accumulative release amounts at each time point, $\mathrm{Cn}$ was the measured concentration at each time point, $\mathrm{V}_{0}$ was the bulk volume of release medium, $V_{i}$ was the sampling volume, $\mathrm{C}_{\mathrm{i}}$ was the measured concentration at time point $i, \mathrm{~W}$ was the total drug amounts in capsules

\section{Release consistence analysis of dissolution in different} medium

The in vitro release feature of minocycline hydrochloride in developed formulation and marketed product was assessed, both the samples were both taken for dissolution determination in hydrochloric acid solution $(0.1 \mathrm{M} \mathrm{HCl})$, purified water, $\mathrm{pH} 4.5$ buffer and $\mathrm{pH} 6.8$ medium. The solution samples were taken for dissolution determination at $0.5,1,2,3,4$ hours. Filtrate was taken to determine the accumulative release amounts at each time point and draw the release curve.

\section{Drug release study and statistical analysis for release data}

According to the sustained-release capsule assay provided by dissolution methods for drug products and the characteristic of product, $0.1 \mathrm{~mol} / \mathrm{L}$ hydrochloric acid solution was used as release medium. Sampling time was reset at $0.5,1,2,3$ and 4 hours.

According to the guideline for bioavailability and bioequiavailability of orally administered solid drugs, similarity $\left(f_{2}\right)$ measuring was applied to evaluate the closeness between the two dissolution profiles. The $f_{2}$ was calculated according to the equations given below:

$f_{2}=50 \times \lg [(1+Q / n)-1 / 2 \times 100] \quad Q=\sum_{t=1}^{n} \quad\left(R_{t}-T_{t}\right) 2$

Where, $\mathrm{n}$ is the number of time points, $R_{t}$ and $T_{t}$ are the percentages of the reference and testing drug release at each time point $t$, respectively

In order to consider the release profiles similar, the $f_{2}$ values should be close to 100 . In general, $f_{2}$ value of the two drug release profiles is between 50 and 100, and then these two drug release characteristics are similar, whereas value below 50 indicates differences between the release profiles.

\section{Results}

\section{Method validation}

Specificity: It was indicated by UV scanning that no interferences from pharmaceutical necessities and solvent were observed. Thus this method showed good specificity and selectivity for the following study under the selected conditions.

Linearity: The calibration curves were prepared at the concentration levels of 3.814-45.768 $\mu \mathrm{g} / \mathrm{mL}$. The typical curve equations were constructed with a weight of $1 / x^{2}$ and described as $\mathrm{A}=0.0341 \mathrm{C}-0.001$, with the correlation coefficients $(r)$ higher than 0.999 .

Precision: The results were assessed at low, median and high levels. The mean RSD determined for intra- and inter-day precision were within $1 \%$.

Recovery: Absolute recovery of $\mathrm{MH}$ was determined by comparing the samples of three levels that incorporated with excipients to that of the standard solutions which were directly diluted by release medium. The recovery were $99.65 \pm 0.54 \%, 99.36 \pm 0.46 \%$ and $99.89 \pm$ $0.76 \%$ at the three concentrations. All these results showed that the absolute recoveries were high enough for the analysis of in preparation.

Stability: The room temperature stability results of $\mathrm{MH}$ with the RSD values lower than $5 \%$ showed that the testing samples were stable under storage conditions and routine analysis for release study.

\section{Release results in reference sample}

The release curve of the reference formulations minocycline hydrochloride extended-release tablets (batch: 701550, $90 \mathrm{mg}$, produced by Barr Laboratories, Inc.) was shown in Figure 1. Contrast results of release rates were summarized in Table I. We can conclude that minocycline hydrochloride in different batches of samples was released with nearly the same property, and release rates increased continuously along with the time.

From the above results, it showed that drug release amounts for each capsule were about $30 \%$ and $50 \%$ at 0.5 and 1 hour, higher than $80 \%$ corresponding to 3 hours. Thus the release rates were set as $20-40 \%, 40-60 \%$ and higher than $80 \%$ for $0.5,1$ and 3 hours.

\section{Release consistence of dissolution in different medium}

The release curves of the developed formulation and marketed product were shown in Figure 2 and Figure 3.

It was indicated that although the release speeds of developed formulation and marketed product were different, almost the total drugs were released from both the preparations at 4 hours. The drug release was delayed about 1 hour and consistent with the gastric emptying time, thus $0.1 \mathrm{M} \mathrm{HCl}$ was selected as the release medium.

\section{Drug release study for sustained-release capsule}

The dissolution curve of developed formulation in 12 batches in hydrochloric acid solution was shown in Figure 4 . The similarity factors $\left(f_{2}\right)$ were calculated for the formulations using the release profile with the marketed product as the reference, the results were shown in Table I. From the above results, the $f_{2}$ values of 12 batches were all within 50-100, suggesting that their release profiles were quite similar to that of the reference. The time points for sampling were set as 1 and 3 hours finally. The release rates results were 


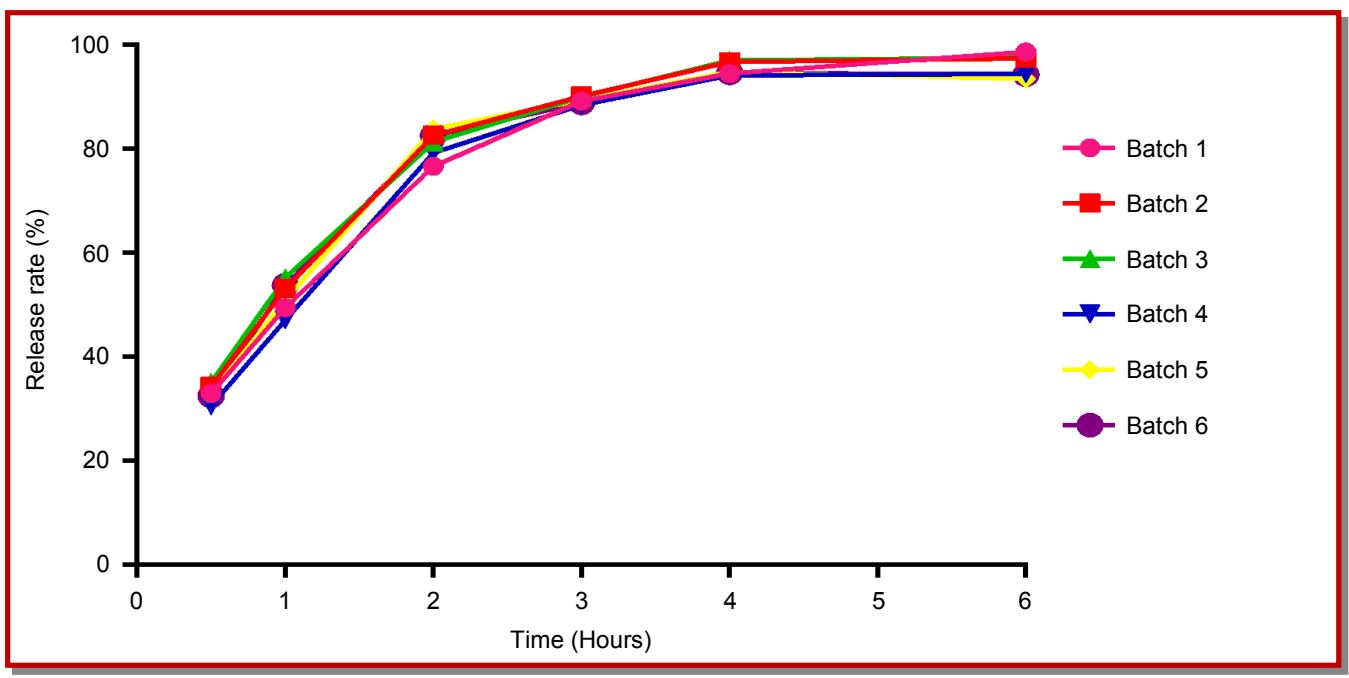

Figure 1: The release curves of the marketed reference sustained-release samples of different batches, with $0.1 \mathrm{M} \mathrm{HCl}$ as the release medium at the rotation speed of $100 \mathrm{rpm}$

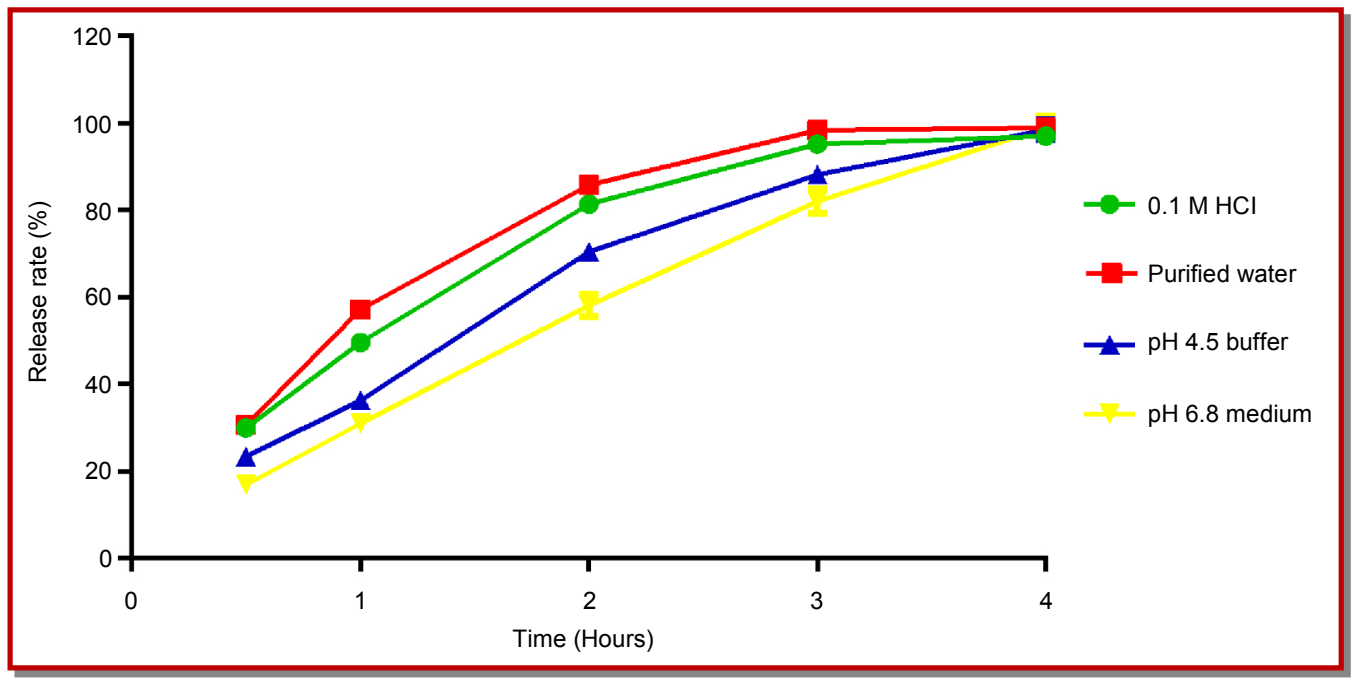

Figure 2: Effects of different mediums on the release of minocycline hydrochloride in marketed product. Each point represents average \pm standard deviation $(n=6)$

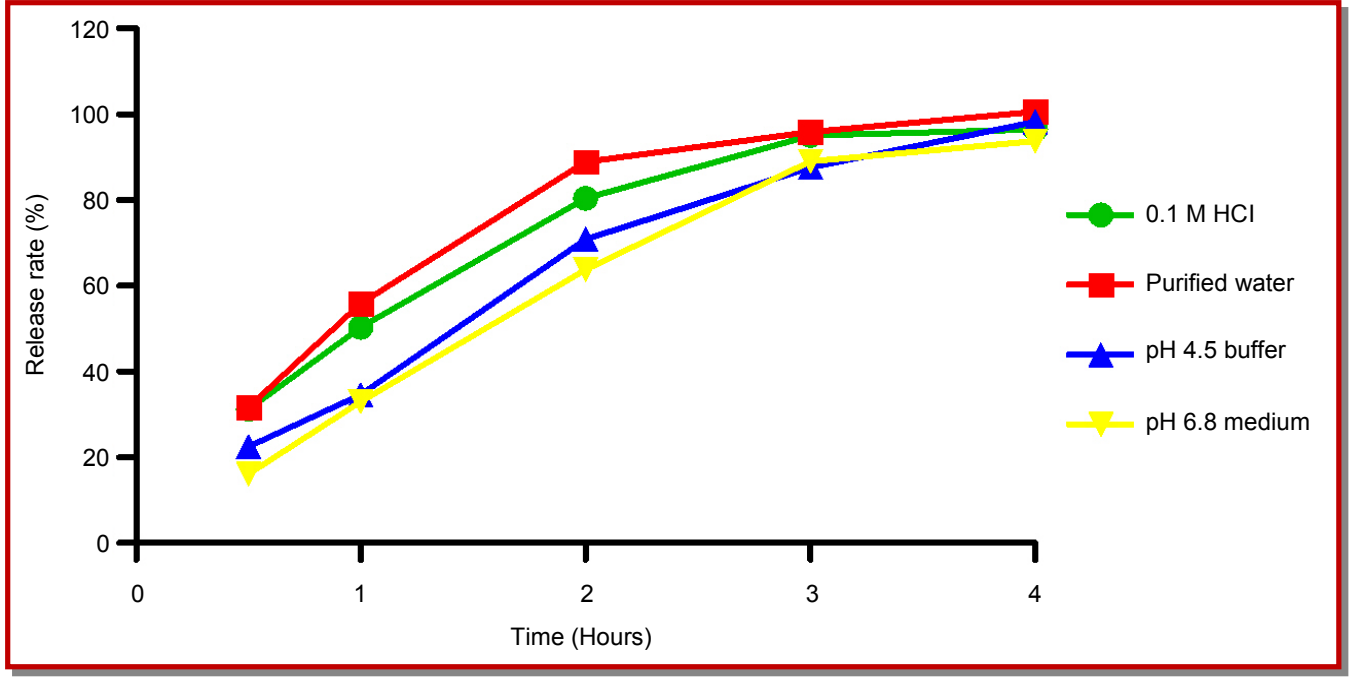

Figure 3: Effects of different mediums on the release of minocycline hydrochloride in developed formulation. Each point represents average \pm standard deviation $(n=6)$ 


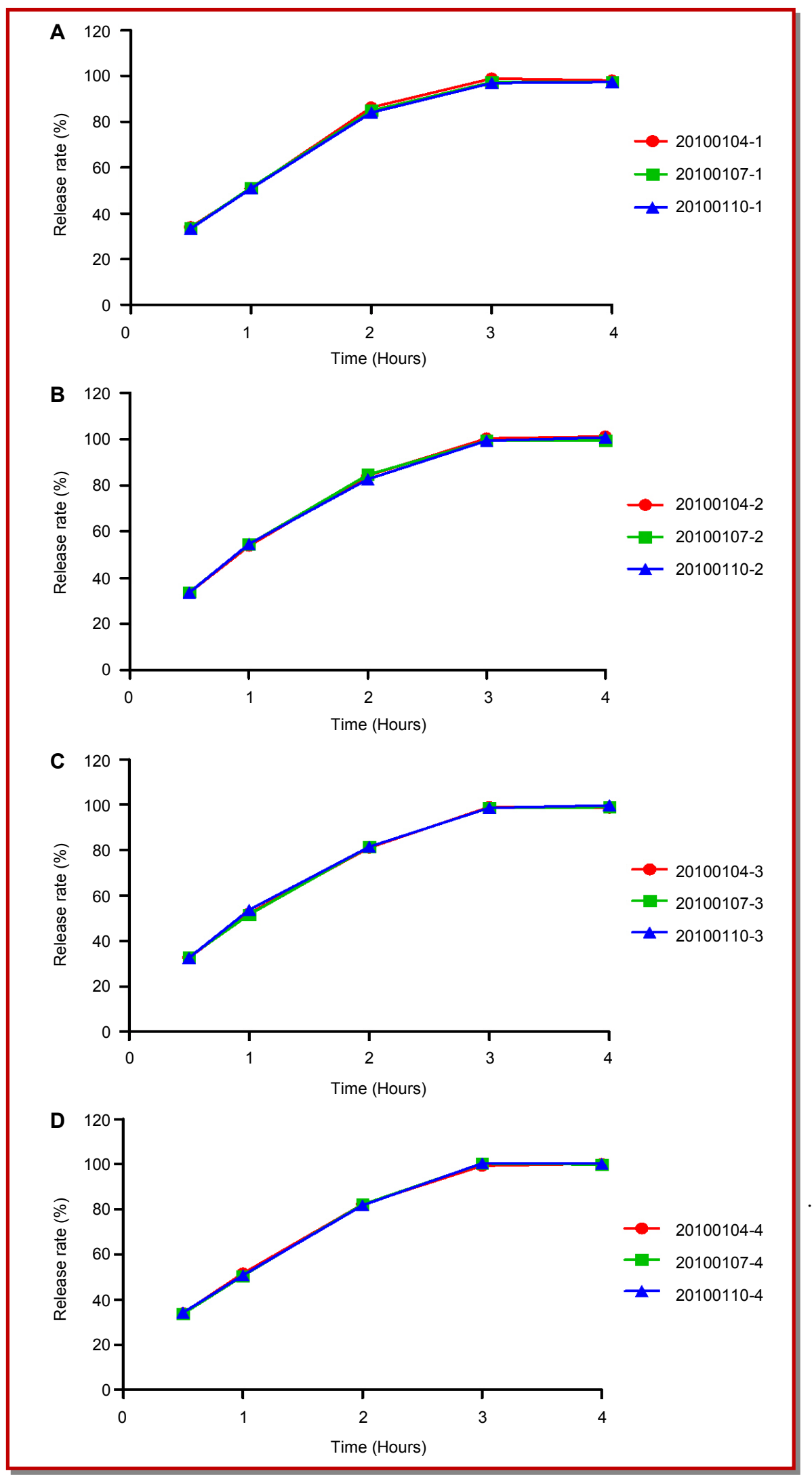

Figure 4: The release curves of the sustained-release capsules in different standard, A: 45 mg, B: 65 mg, C: 90 mg, D: 115 mg. Each point represents average \pm standard deviation $(n=6)$ 
Table I

\begin{tabular}{|c|c|c|c|}
\hline \\
\hline \multicolumn{4}{|c|}{$\begin{array}{l}\text { The similarity factors (f2) calculating results for } \\
\text { sustained-release capsules in different batches } \\
\text { Batch }\end{array}$} \\
\hline \multicolumn{4}{|c|}{ 20100107-1 } \\
\hline 20100110-1 & 74.76752 & 20100104-2 & 64.19677 \\
\hline 20100110-2 & 66.41798 & $20100107-2$ & 66.32182 \\
\hline 20100104-3 & 72.33353 & 20100107-3 & 72.87776 \\
\hline 20100110-3 & 70.10086 & 20100104-4 & 69.17828 \\
\hline 20100107-4 & 68.33718 & 20100110-4 & 67.37129 \\
\hline
\end{tabular}

Table II

In vitro release experimental results in $0.1 \mathrm{M} \mathrm{HCl}$

\begin{tabular}{|c|c|c|}
\hline Batch & 1 hour & 3 hours \\
\hline 20100104-1 & 50.80 & 98.81 \\
\hline 20100107-1 & 51.21 & 97.33 \\
\hline 20100110-1 & 50.88 & 96.88 \\
\hline 20100104-2 & 53.76 & 100.29 \\
\hline 20100107-2 & 54.45 & 99.26 \\
\hline 20100110-2 & 54.56 & 99.24 \\
\hline 20100104-3 & 52.24 & 99.08 \\
\hline 20100107-3 & 51.65 & 98.50 \\
\hline 20100110-3 & 53.75 & 98.54 \\
\hline 20100104-4 & 51.56 & 99.26 \\
\hline 20100107-4 & 50.59 & 100.34 \\
\hline 20100110-4 & 50.78 & 100.47 \\
\hline Reference & 50.28 & 95.04 \\
\hline
\end{tabular}

summarized in Table II.

\section{Discussion}

According to the sustained-release capsule assay provided by dissolution methods for drug products and the characteristic of product (Tsao et al., 2014; Chopra et al., 2015), $0.1 \mathrm{M} \mathrm{HCl}$ solution was selected as release medium, $100 \mathrm{rpm}$ was selected as rotation speed. To make the release curve more precisely, 0.5 and 3 hours were added as the time points for sampling, thus the sampling was defined at 0.5, 1, 2, 3 and 4 hours (Peng et al., 2014; Xue et al., 2014).

For the selection of detection wavelength, the testing solution was taken for UV scanning at 200-600 nm, the result showed that the maximum absorption of $\mathrm{MH}$ was at $348 \mathrm{~nm}$, with the absorption value 0.9825 . According to the guidelines for UV-spectrophotometric method in Chinese Pharmacopoeia, 2000 edition, the appropriate UV absorption for testing solution was 0.30.7 (Simpson et al, 2014; Ifuku et al, 2014), thus $348 \mathrm{~nm}$ was set as the detection wavelength. The detection concentration was set as $15 \mu \mathrm{g} / \mathrm{mL}\left(\mathrm{C}_{23} \mathrm{H}_{27} \mathrm{~N}_{3} \mathrm{O}_{7}\right)$.

\section{Conclusion}

We prepared minocycline hydrochloride sustainedrelease capsules using the new extrusion-spheronization preparative method, and developed an analysis method for the quantification and dissolution study of $\mathrm{MH}$. The results showed this assay method was reliable and convenient enough for the rapid determination of $\mathrm{MH}$ contents quantitatively in high-throughput release characteristic studies. Furthermore, the drugs could be well released from sustained-release carriers within the specified time limit, and the release characteristics of the developed formulation and commercial samples were quite consistent with each other.

\section{References}

Blanchard C, Barnett P, Perlmutter J, Dunman PM. Identification of Acinetobacter baumannii serum-associated antibiotic efflux pump inhibitors. Antimicrob Agents Chemother. 2014; 58: 6360-70.

Bunagan MJ, Banka N, Shapiro J. Retrospective review of folliculitis decalvans in 23 patients with course and treatment analysis of long-standing cases. J Cutan Med Surg. 2015; 19: 45-49.

Chopra S, Harjai K, Chhibber S. Antibiotic susceptibility of icapositive and ica-negative MRSA in different phases of biofilm growth. J Antibiot (Tokyo). 2015; 68: 15-22.

Cui L, Peng WX, Sun ZJ, Lu HF, Chen GN. Variability of macroscopic dimensions of Moso bamboo. Pakistan J Pharmaceut Sci. 2015; 28: 675-79.

Dong X, Chen F, Zhang Y, Liu H, Liu Y, Ma L. In vitro activities of rifampin, colistin, sulbactam and tigecycline tested alone and in combination against extensively drug-resistant Acinetobacter baumannii. J Antibiot (Tokyo). 2014; 67: 67780.

Freiberg S, Zhu XX. Polymer microspheres for controlled drug release. Int J Pharm. 2004; 282: 1-18.

Ifuku M, Hossain SM, Noda M, Katafuchi T. Induction of interleukin- $1 \beta$ by activated microglia is a prerequisite for immunologically induced fatigue. Eur J Neurosci. 2014; 40: 3253-63.

Li YH, Zhu JB. Modulation of combined-release behaviors from a novel "tablets-in-capsule system". J Control Release. 2004; 95: 381-89.

Liu Y, Sun YH, Sun J, Zhao NN, Sun MY, He ZG. Preparation and in vitro/in vivo evaluation of sustained-release venlafaxine hydrochloride pellets. Int J Pharm. 2012; 426: 2128.

Nagpal K, Singh SK, Mishra DN. Minocycline encapsulated 
chitosan nanoparticles for central antinociceptive activity. Int J Biol Macromol. 2015; 72: 131-35.

Norden DM, Bicer S, Clark Y, Jing R, Henry CJ, Wold LE, Reiser PJ, Godbout JP, McCarthy DO (2015). Tumor growth increases neuroinflammation, fatigue and depressive-like behavior prior to alterations in muscle function. Brain Behav Immun. 2015; 43: 76-85.

Oya K, Kishi T, Iwata N. Efficacy and tolerability of minocycline augmentation therapy in schizophrenia: A systematic review and meta-analysis of randomized controlled trials. Hum Psychopharmacol. 2014; 29: 483-91.

Peng WX, Lin $\mathrm{Z}$, Chen $\mathrm{H}$, Wu JG. Biochemical group characteristics of self-bonded boards during acidic oxidation for public health. J Pure Appl Microbiol. 2015; 9: 307-11.

Peng WX, Wang LS, Zhang ML, Lin Z. Separation characteristics of lignin from Eucalyptus camaldulensis lignincelluloses for biomedical cellulose. Pakistan J Pharmaceut Sci. 2014; 27: 723-28.

Ross AC, Macrae R. Chronopharmaceutical drug delivery from a pulsatile capsule device based on programmable erosion. J
Pharm Pharmacol. 2000; 52: 903-09.

Shibata N, Nishumura A, Naruhashi K, Nakao Y, Miura R. Preparation and pharmaceutical evaluation of new sustained -release capsule including starch-sponge matrix (SSM). Biomed Pharmacothr. 2010; 64: 352-58

Simpson B, Foster S, Ku JH, Simpson EL, Ehst BD. Triple antibiotic combination therapy may improve but not resolve granuloma annulare. Dermatol Ther. 2014; 27: 343-47.

Tsao C, McGoey RR. Radiology case of the month: 49-year-old female with an anterior mediastinal mass. J La State Med Soc. $2014 ; 166$ : 134-36.

Vargiu AV, Ruggerone P, Opperman TJ, Nguyen ST, Nikaido H. Molecular mechanism of MBX2319 inhibition of Escherichia coli AcrB multidrug efflux pump and comparison with other inhibitors. Antimicrob Agents Chemother. 2014; 58: 6224-34.

Xue Q, Peng WX, Ohkoshi M. Molecular bonding characteristics of self-plasticized bamboo composites. Pakistan J Pharmaceut Sci. 2014; 27: 975-82.

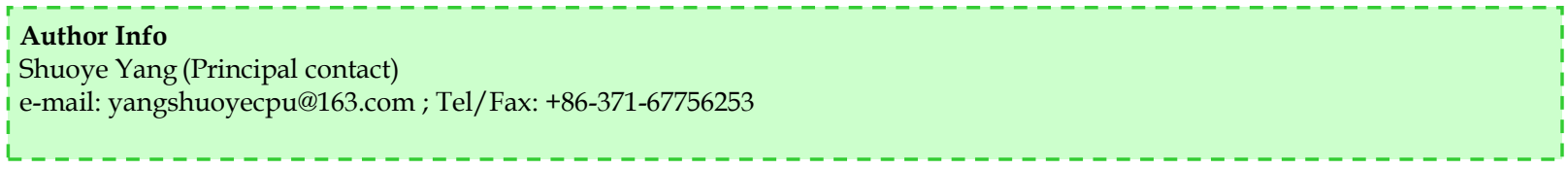




\section{Your feedback about this paper}

1. Number of times you have read this paper 0

2. Quality of paper
Excellent
Good
Moderate
Not good

3. Your comments

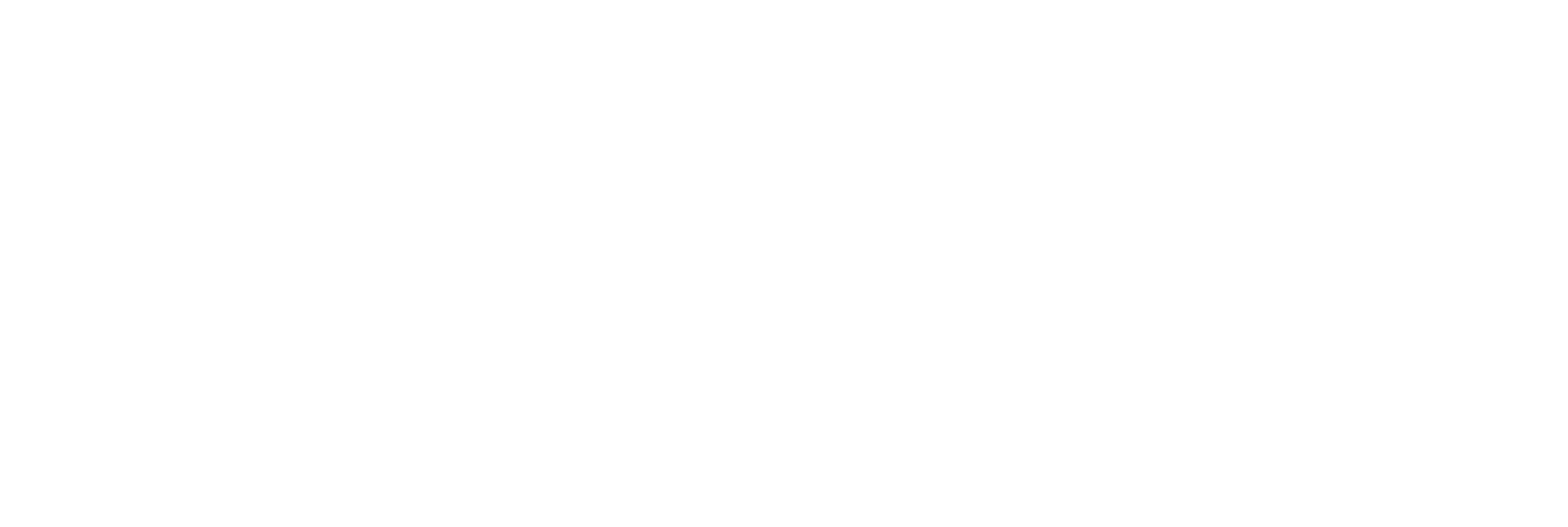

